\title{
Physique du sédiment et des eaux interstitielles : stratégies de modélisation pour les besoins en environnement côtier
}

\author{
Physics of sediment and pore-water: modelling strategies for Coastal \\ Environment studies \\ PIERRE LE HIR \\ IFREMER \\ Centre de Brest, laboratoire DYNECO/PHYSED, BP 70, 29280 Plouzané, France \\ Tél. : +33 (0)2 982243 40, Fax : +33 (0)2 982248 64, e-mail : plehir@ifremer.fr
}

\section{A} fter a brief reminder on the sediment composition and facies, the paper reviews water-sediment exchanges that require to account for sediment transformation in Coastal Environment modelling. Physical processes (consolidation, bed armouring, liquefaction) and bio-chemical processes (organic matter mineralisation, adsorption/desorption, precipitation/dissolution, bioturbation...) are briefly reviewed, as well as usual modelling methods. The need for a guide book on the required minimal modelling, depending on the arisen questions, is put forward.

\section{I INTRODUCTION}

C'est un lieu commun de dire que le sédiment joue un rôle fondamental dans la santé des écosystèmes côtiers, et leur évolution à long terme. Cependant, rares sont les modélisations d'écosystèmes prenant en compte l'ensemble des processus qui interviennent au sein du sédiment, de telle sorte qu'en général chaque entreprise de modélisation du sédiment n'aborde ce compartiment que sous un angle donné, pour des processus particuliers. Cette publication vise à parcourir les différentes approches, à les classer, et à dresser les caractéristiques d'un modèle de sédiment qui réponde aux principales questions posées en environnement côtier.

Dans une première partie, un rappel est fait sur la composition des sédiments marins (côtiers), leurs conditions de formation et leur structuration verticale ou horizontale.

Une seconde partie présente les processus qui motivent la prise en compte du sédiment dans les modèles d'environnement côtier. Il s'agit en général de suivre l'évolution spatio-temporelle de la nature du sédiment et de quantifier les échanges à l'interface eau/sédiment : échanges particulaires par érosion/dépôt ou par bioturbation, échanges d'éléments dissous (également par érosion/dépôt, mais aussi par diffusion et bioturbation).

Puis un inventaire des processus au sein du sédiment est dressé, et les principales approches de modélisation sont indiquées, en mettant l'accent sur les processus physiques : consolidation, liquéfaction, diffusion...

En quatrième partie, des exemples de modélisation couplée eau/sédiment sont décrits brièvement.

\section{II $\square$ RAPPELS SUR LA COMPOSITION DES SÉDIMENTS}

De façon très schématique, un sédiment est constitué d'un réseau de particules minérales (essentiellement des grains de quartz ou de feldspath, des carbonates et des particules argileuses très fines) et de matière organique. Différents composés chimiques sont présents, soit sous forme dissoute dans l'eau interstitielle, soit sous forme adsorbée sur les particules. Les particules sont fréquemment classées par leur taille, dont l'échelle varie entre le $\mu \mathrm{m}$ et le $\mathrm{dm}$.

Lorsque les particules «grossières » dominent (typiquement plus de 50-100 $\mu \mathrm{m}$ ), les forces d'interaction entre grains sont faibles, le sédiment est dit non-cohésif. En fonction des conditions de dépôt ou de remaniement par les vagues, les arrangements entre grains peuvent varier, influençant alors les conditions de leur mise en mouvement : la porosité (fraction volumique des espaces entre grains) en est l'indicateur le plus simple, et constitue le paramètre descripteur essentiel du sédiment, complémentaire de sa densité et du spectre granulométrique. Dans un environnement où le dépôt domine, l'arrangement entre les grains est lâche (porosité de 40-45\%) tandis que sous l'action des vagues le sédiment se densifie (porosité de 30-35\%) et les grains sont plus difficiles à remanier. A noter que la porosité d'un sédiment hétérométrique est plus faible puisque des petits grains peuvent se placer entre les plus gros.

Dans le cas des vases, le sédiment est composé d'une majorité de particules fines (argiles $<2-5 \mu$, silts $<63 \mu$ ): les distances inter-particulaires diminuent et les forces d'interaction entre grains deviennent importantes. Ce sont soit des forces d'attraction (par exemple les forces de Van der Waals), soit des forces de répulsion (liées aux charges électriques), dont l'intensité dépend de la concentration cationique du milieu et de la distance entre les particules. Selon leur importance respective, il en résulte un état stable de dispersion ou d'agglomération, comme le rappelle la figure 1. Le sédiment devient cohésif. C'est surtout la teneur en argiles qui détermine les propriétés de cohésion, mais l'incertitude sur leur évaluation conduit à considérer la teneur en fines $(<$ $63 \mu$ ), plus accessible (par exemple par filtration). Un dépôt frais de sédiment cohésif peut se compacter sous son propre poids, et la consolidation qui en résulte modifie son comportement à l'érosion. 


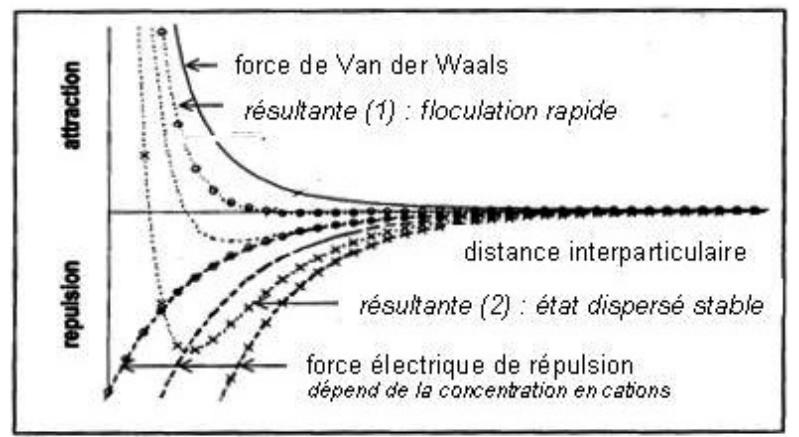

Figure 1 : représentation schématique des forces d'attraction et de répulsion entre particules d'argile, en fonction de la concentration cationique

(d'après Winterwerp et van Kesteren, 2004 [14]).

Un sédiment naturel peut aussi être constitué d'un mélange de vases et de particules sableuses, dont le comportement est mal connu et fait actuellement l'objet de nombreuses recherches. Selon Van Ledden et al. [13], le diagramme triangulaire dans lequel la texture du sédiment est représentée par sa teneur en sable, en silt et en argile (fig. 2) constitue un mode de représentation pertinent du sédiment, la teneur en argile déterminant le comportement cohésif tandis que des teneurs élevées en sable (resp. en silt) indiquent une probable structuration sableuse (resp. silteuse) du sédiment selon la porosité.

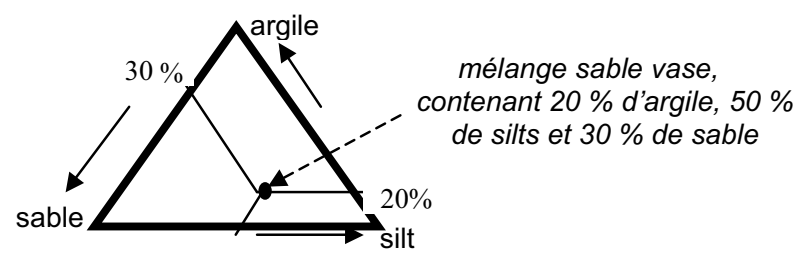

Figure 2 : diagramme textural triangulaire.

La combinaison de sables et de vases peut aussi se présenter sous forme de litages, constitués lors d'épisodes de dépôts successifs ou résultant d'une sédimentation différentielle. Ils peuvent être la signature d'événements très courts.
Enfin, les faciès sédimentaires présentent souvent de fortes hétérogénéités spatiales (Fig.3), liées à la nature du sédiment, mais aussi aux reliefs (chenaux d'écoulement, rides, ondulations sableuses, cratères et autres macrorugosités), à la présence d'organismes (bactéries et microphytobenthos, végétaux, meiofaune et macrofaune benthique, poissons...). Ces faciès sont typiquement distribués selon des échelles variant entre le $\mathrm{dm}$ et le $\mathrm{km}$.

\section{III — POURQUOI INTÉGRER LE SÉDIMENT DANS UNE MODÉLISATION CÔTIÈRE?}

Quel que soit le domaine scientifique auquel s'applique une modélisation du milieu côtier, le compartiment sédiment doit être pris en compte de façon plus ou moins explicite. Ainsi même en hydrodynamique, la nature du sédiment intervient par la rugosité de l'interface (rugosité de grain et rugosité de forme). Parfois, le mouvement du sédiment accentue l'effet de frottement perçu par la colonne d'eau: c'est le cas au-dessus d'un sable charrié, ou le long des littoraux vaseux exposés, près desquels les houles du large sont largement amorties par la liquéfaction du sédiment.

\section{III.1 BESOINS DE DESCRIPTION DU SÉDIMENT POUR LA DYNAMIQUE SÉDIMENTAIRE}

La stratégie de modélisation des transports sédimentaires diffère selon leur mode de transport. Pour les sédiments grossiers dont la vitesse de chute est élevée et qui sont transportés par charriage pur ou à proximité du fond (saltation), une hypothèse de transport à l'équilibre est souvent formulée (parfois avec un terme de relaxation), en fonction de la granulométrie du fond. Le module "sédiment» doit alors gérer la disponibilité en sédiment pour chaque classe et rendre compte des érosions/dépôts calculés à partir de la divergence des capacités de transport. Le cas des sédiments non cohésifs hétérométriques nécessite la prise en compte de processus de masquage/exposition (protection de petits grains à l'abri d'éléments grossiers, mise en mouvement facilitée de ces derniers lorsqu'ils sont environnés de particules plus mobiles) et du processus de pavage : ce dernier traduit la limitation du transport lorsque les particules les plus fines du sédiment superficiel ont été érodées et qu'une carapace de sédiment grossier moins mobile protège en surface les grains plus fins enfouis. La difficulté majeure pour simuler

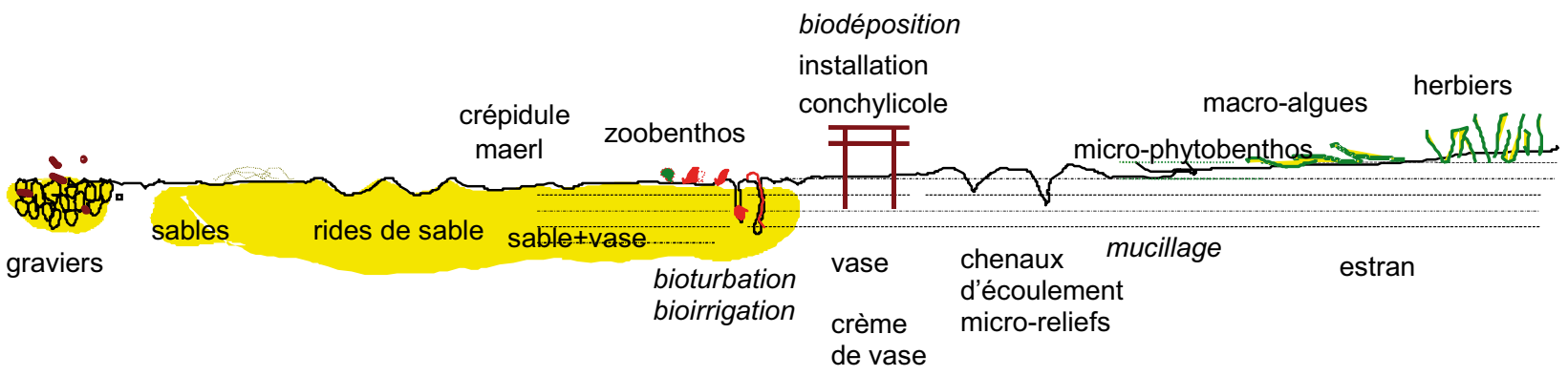

Figure 3 : illustration de la variabilité des facies sédimentaires naturels ou anthropisés. 
le processus est la détermination de l'épaisseur de la couche active, celle sur laquelle les remaniements de grains peuvent se produire. Cette épaisseur varie selon les auteurs en fonction de la dimension des éléments les plus gros et/ou de l'intensité du forçage hydrodynamique.

Dans le cas des sédiments cohésifs caractérisés par une faible vitesse de chute, le transport en suspension domine, et les mouvements des particules sont calculés en résolvant une équation d'advection/diffusion dont les termes sources et puits sont les flux d'érosion et de dépôt. Ces échanges doivent être pris en compte pour gérer la disponibilité des matériaux dans le sédiment. En outre, les flux d'érosion dépendent de l'érodabilité du sédiment, qui elle-même varie en fonction de la nature du sédiment et de son état de consolidation : le processus de tassement doit donc être considéré dans le modèle sédiment.

Si le sédiment est constitué d'un mélange cohésif / non cohésif, la tendance actuelle consiste à formuler l'érosion pour le mélange lui-même, et résoudre une équation d'advection/diffusion pour chaque classe de particules, en considérant que le transport se fait en suspension ou en saltation (e.g. Waeles et al., 2007 [15]). Cependant, pour la fraction grossière transportée en charriage pur, il peut être nécessaire de recourir à une formulation de capacité de transport (rarement proposée dans la littérature pour un sédiment mélangé), et d'ajouter aux érosions/dépôts calculés pour les sédiments transportés en suspension un échange à l'interface de sédiments grossiers déduit de la divergence des capacités de transport.

L'érodabilité des sédiments mixtes sable/vase est un objet d'études récent. Les expériences montrent qu'en dessous d'une fraction critique de particules fines, le sédiment se comporte comme un sédiment non cohésif, avec un seuil d'érosion faible, et qu'au delà de cette fraction critique, un effet de cohésion apparaît et la contrainte seuil d'érosion augmente avec la fraction fine (Panagiotopoulos et al., 1997 [8]), tout en restant dépendante de l'état de consolidation (Le Hir et al., 2006 [4]). A noter que dans le cas d'un sable pur, des mesures en canal d'érodimétrie montrent que le seuil de remaniement, classiquement exprimé par le diagramme de Shields, est fortement accru lorsque la porosité du sable est réduite par tassement, ce que peuvent provoquer des vagues répétées.

La présence de végétaux est susceptible de modifier les échanges particulaires à l'interface : la canopée réduit la contrainte de cisaillement sur le fond, tandis que les racines renforcent la rigidité du sédiment, les deux mécanismes atténuant l'érosion. Le microphytobenthos (diatomées benthiques), souvent répandu sur les estrans vaseux exposés à la lumière, modifie aussi la résistance à l'érosion du sédiment superficiel en sécrétant du mucus qui en augmente la cohésion. Enfin la faune benthique participe aux échanges particulaires à l'interface, soit directement en ingérant des matières en suspensions (suspensivores) ou en rejetant des feces et pseudofeces, soit indirectement en modifiant la rugosité du fond ou en altérant les propriétés physiques du sédiment, par bioturbation. Tous ces facteurs biologiques, qui caractérisent le sédiment, devraient donc être pris en considération dans une modélisation des transports de sédiments dans un environnement naturel, ce qui est pourtant rarement le cas (Le Hir et al., 2007 [6]).

\section{III.2 BESOINS DE DESCRIPTION DU SÉDIMENT POUR LA CHIMIE ET LA BIOCHIMIE}

Que la finalité soit la contamination chimique en mer côtière ou la caractérisation des processus de transformation de la matière organique et le cycle des éléments nutritifs, l'étude du devenir des éléments chimiques qui ont une forme particulaire ne peut ignorer le sédiment. Les échanges entre la colonne d'eau et le sédiment sont alors un sujet sensible, car les temps de séjour dans le milieu marin des contaminants ou des éléments naturels tels que les nutriments peuvent être considérablement modifiés, par piégeage plus ou moins temporaire dans le sédiment et relargage ultérieur. Les échanges d'éléments chimiques se produisent par érosion/dépôt, mais aussi sous forme dissoute car un contaminant particulaire est susceptible de se désorber au sein du sédiment. Plus généralement, des processus diagénétiques prennent place dans le sédiment, engendrant des flux d'éléments dissous (contaminants, nutriments...), soit par diffusion moléculaire, soit par diffusion turbulente à l'interface sous l'effet du courant et/ou des vagues, soit par transfert d'eau interstitielle sous l'effet du tassement, ou encore sous l'effet de gradients de pression (cas des fonds sableux ridés perméables, près de l'interface). Les expressions de ces différents termes d'échanges dissous à l'interface sont en particulier répertoriées dans l'ouvrage de synthèse de Boudreau (1997) [1]. Les transformations dans le sédiment sont quant à elles évoquées dans le $\S \mathrm{IV}$ ci-après.

\section{III.3 BESOINS DE DESCRIPTION DU SÉDIMENT POUR L'ÉCOLOGIE BENTHIQUE}

Le développement de la flore benthique (herbiers, algues ou microphytobenthos) comme celui de la faune benthique est naturellement dépendant de la nature du sédiment superficiel. C'est en particulier la fonction d'habitat qu'il nous faut préciser, car leur préservation devient une préoccupation grandissante pour la durabilité des écosystèmes.

Le conjonction de la sensibilité des transferts particulaires à l'occupation du sédiment par la flore ou la faune benthique et de la dépendance des développements biologiques en fonction de la nature du fond et des forçages hydrodynamiques devrait conduire dans un avenir proche à des modélisations couplées physique/biologie.

\section{IV — PROCESSUS AU SEIN DU SÉDIMENT}

Le sédiment est le siège de transformations diverses à différentes échelles de temps. On s'intéresse ici à la diagénèse précoce qui recouvre l'ensemble des processus de transformation à " courte » échelle de temps (au sens géologique). Classiquement cependant, on identifie d'abord des transformations physiques du sédiment, essentiellement le tassement et la liquéfaction, en réservant le terme de transformation diagénétique aux processus physico-chimiques au sein du sédiment (minéralisation, adsorption/désorption et diffusion). 


\section{IV.1 SÉDIMENTATION, TASSEMENT ET CONSOLIDATION}

Un dépôt frais de sédiment fin est en général constitué de flocs et se caractérise par une porosité élevée. Une première phase de tassement commence alors, contrôlée par la perméabilité du sédiment et l'excès de pression interstitielle. Pendant ce processus, il peut y avoir ségrégation de la fraction sableuse par décantation différentielle, tant que le sédiment reste peu dense. A partir d'une certaine concentration (proche de la notion de concentration de gel), les contacts entre particules se généralisent et permettent une transmission «grain à grain » des contraintes dans le sédiment : ce sont les contraintes effectives, qui ont pour effet de réduire l'excès de pression interstitielle et donc l'évacuation de l'eau interstitielle. C'est la deuxième phase de consolidation, dont la fin intervient lorsque les excès de pression interstitielle sont totalement dissipés. Ces deux premières phases représentent la consolidation primaire, par opposition à la consolidation secondaire (parfois appelée fluage) qui se poursuit, et que l'on peut relier à une déformation du squelette sédimentaire sous l'action de son poids déjaugé. L'ensemble du mécanisme de consolidation primaire est décrit par l'équation de Gibson (1967) qui exprime la variation de la teneur en eau en fonction des profils de perméabilité et de contrainte effective. Cette équation est «fermée» par des relations de constitution qui relient ces deux paramètres à la teneur en eau (ou à la concentration du sédiment). Depuis une quinzaine d'année, plusieurs modèles numériques résolvant l'équation de Gibson ont été proposés pour les vases pures, parmi lesquels on peut mentionner celui de Pham Van Bang et al., 2006 [9] qui utilise une technique numérique de capture de choc pour décrire la propagation verticale des fronts de concentration. Le lien entre sédimentation pure et consolidation est explicité par Toorman (1996) [12], tandis que Merckelbach (2000) [7] a réécrit l'équation de Gibson pour l'étendre au mélange sable/vase. Tout récemment, nous avons développé un modèle de consolidation sur la base de la théorie de Gibson revue par Merckelbach, qui décrit l'évolution de la concentration pour plusieurs classes granulométriques et autorise une sédimentation différentielle en début de consolidation

$$
\frac{\partial C_{i}}{\partial t}=-\frac{\partial}{\partial z}\left[C_{i} \max \left\{\frac{k}{\rho_{w}}\left(C \frac{\rho_{s}-\rho_{w}}{\rho_{s}}+\frac{1}{g} \frac{\partial \sigma^{\prime}}{\partial z}\right), w s_{i, \text { hindered }}\right\}\right]
$$

où $\mathrm{C}_{\mathrm{i}}$ désigne la concentration massique de la classe $\mathrm{i}$, $\mathrm{C}$ la concentration totale, $\mathrm{k}$ la perméabilité du sédiment, $\sigma^{\prime}$ la contrainte effective, $\rho_{\mathrm{s}}$ et $\rho_{\mathrm{w}}$ les densités des grains et de l'eau, g l'accélération de la pesanteur et $\mathrm{ws}_{\mathrm{i} \text {,hindered }}$ la vitesse de chute entravée de la classe i. La perméabilité est exprimée en fonction de la porosité et d'un diamètre représentatif des grains les plus fins. Ce modèle permet de reconstituer le tassement des vases et des mélanges sable/ vase (figure 4).

A partir de la concentration du sédiment ainsi calculée, il est possible de reconstituer son érodabilité en surface en utilisant une formulation empirique calibrée sur des expériences érodimétriques.

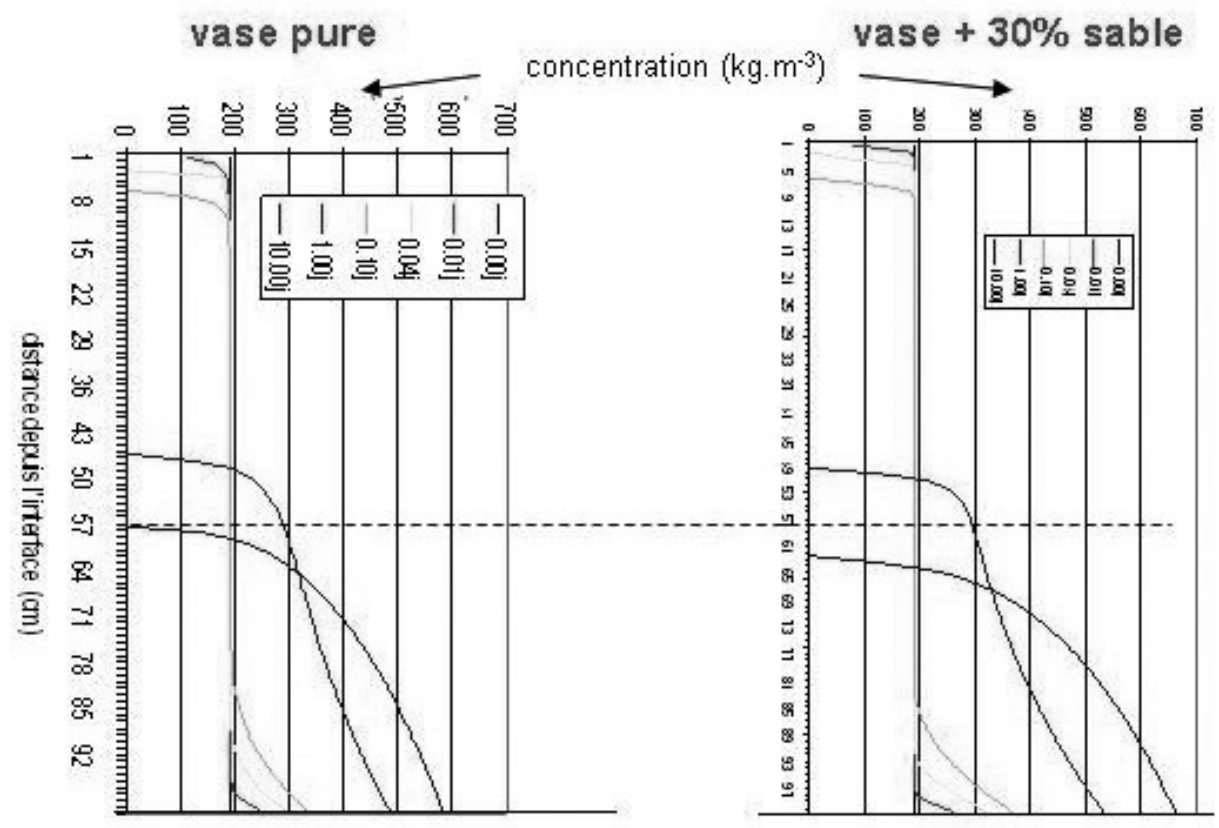

Figure 4 : Simulation de l'évolution du profil de concentration dans une colonne à sédimentation. Mise en évidence d'une accélération du tassement en présence de sable. Les instants des profils sont en jours. 


\section{IV.2 LIQUÉFACTION PAR LES VAGUES}

Lorsque les vagues se propagent sur un fond vaseux, elles peuvent engendrer un mouvement oscillant au sein du sédiment, qui se traduit par un amortissement des vagues et parfois une liquéfaction du sédiment. Le processus de liquéfaction provient d'une altération rhéologique de la vase par réduction rapide de son élasticité et de sa viscosité lorsque l'amplitude de déformation dépasse un certain seuil (Silva Jacinto et Le Hir, 2001 [10]). En couplant les variations des caractéristiques rhéologiques des vases aux déformations générées par les vagues, ces auteurs ont développé un modèle analytique multicouches capable de prédire l'apparition d'une zone liquéfiée en fonction des vagues en surface. Bien qu'a priori considérable, la conséquence de ce mécanisme en termes d'érosion accrue n'a pas encore été complètement quantifiée ; il est intéressant de noter que ce mécanisme de liquéfaction n'entraîne pas de variation de densité du sédiment avant son éventuelle érosion.

\section{IV.3 AUTRES PROCESSUS PHYSIQUES}

D'autres processus physiques interviennent dans le sédiment, en particulier en présence de gaz, souvent lié à la dégradation de matière organique. Un cas particulier important est l'exondation d'un estran à marée basse, qui se traduit par un dessèchement du sédiment superficiel et donc un tassement accéléré, et une érodabilité moindre du sédiment lors de son recouvrement. Cependant, la phase de recouvrement s'accompagne en général d'une réhydratation et d'un gonflement du sédiment, mais qui est susceptible de n'intervenir qu'après passage du courant de flot. Une modélisation empirique de ce phénomène a été proposée par Le Hir et al. (1989)[3].

Les dégagements gazeux sont aussi connus pour favoriser les glissements sous-marins. Plus généralement, un sédiment pentu peut devenir instable et conduire à des écroulements (cas de certains estrans estuariens qui sont soumis à affouillement par l'écoulement à marée basse) ou des glissements (par exemple sur les rebords des plateaux continentaux). Ces phénomènes physiques sont en général traités selon une approche de type mécanique des sols, que nous n'abordons pas ici.

\section{IV.4 PROCESSUS DIAGÉNÉTIQUES}

Les éléments chimiques déposés dans le sédiment subissent de multiples transformations bio-physico-chimiques : des réactions d'adsorption/désorption, de précipitation/dissolution, de minéralisation (pour la matière organique), d'oxydo/réduction se produisent, avec de multiples variables de contrôle telles que les bactéries, le $\mathrm{pH}$, la température, les ions métalliques... et surtout l'oxygène dissous qui pénètre le sédiment superficiel sur quelques $\mathrm{cm}$. Les modèles doivent donc mettre en œuvre des équations en général couplées selon une discrétisation verticale plus ou moins fine. La figure 5 ci-dessous illustre la diversité des échanges à considérer pour seulement un élément, le phosphore, sans en montrer la complexité. Cependant des simplifications sont souvent pertinentes, et le biochimiste conserve l'initiative de sélectionner les réactions qu'il juge prédominantes dans un

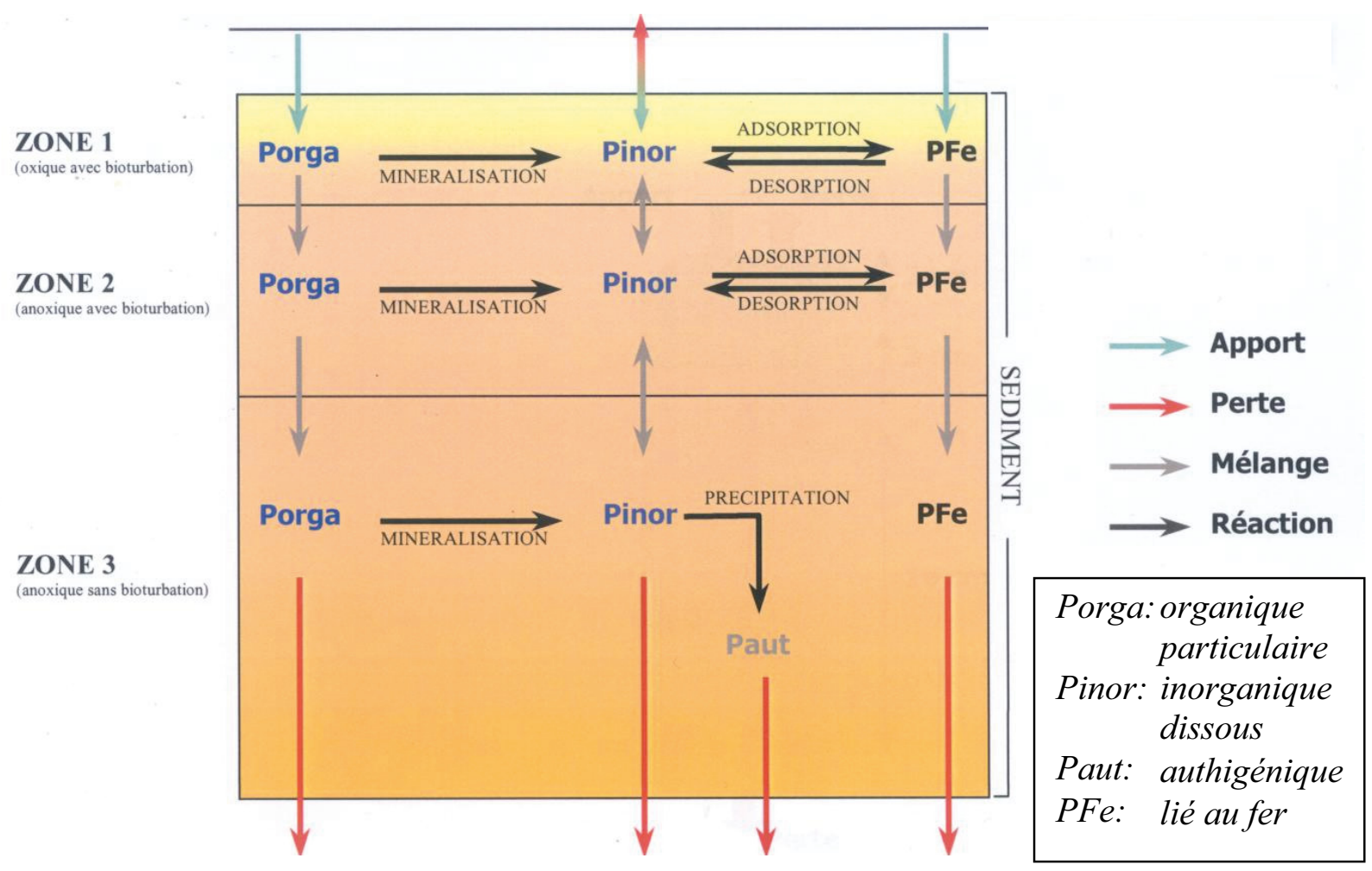

Figure 5 : exemple de modèle diagénétique développé pour le phosphore (Andrieux, com. pers., d'après Slomp et al., 1996 [11]). 
environnement donné : ce sont donc en général des modèles génériques de diagénèse qui sont développés, le choix des processus à simuler relevant des applications.

Des stratégies similaires sont adoptées en modélisation écologique. Par exemple, le modèle Elise de production primaire, appliqué à la baie de Seine par Cugier et al. (2005) [2] considère le devenir dans le sédiment de trois éléments naturels majeurs, $\mathrm{P}, \mathrm{N}$ et $\mathrm{Si}$, apportés par décantation de matériel détritique ou par dépôt de diatomées, puis transformés en éléments dissous après mortalité des diatomées dans le sédiment et minéralisation du matériel détritique, et enfin échangés avec l'eau surnageante par diffusion.

\section{IV.5 DIFFUSION DANS L'EAU INTERSTITIELLE}

Le processus de diffusion dans l'eau interstitielle contrôle les transferts verticaux d'éléments dissous : c'est lui qui permet leur remontée vers la surface, en complément du transport direct par l'eau interstitielle lors de la consolidation ou par biodiffusion (cf $\S$ IV.6). En général le flux diffusif est évalué à partir du gradient vertical de concentration (loi de Fick), en introduisant un coefficient de diffusion " effectif»" qui rend compte du cheminement complexe que les composés dissous doivent effectuer, paramétré par la tortuosité q. Selon Boudreau (1997) [1] :

$$
\begin{gathered}
\mathrm{D}^{\prime}=\mathrm{D} / \theta^{2}, \mathrm{D}(\text { diffusion moléculaire }) \sim 10^{-8} \mathrm{~m}^{2} \cdot \mathrm{s}^{-1} \\
\text { avec } \theta^{2} \sim 1-\ln \left(\mathrm{n}^{2}\right) \text { où } \mathrm{n} \text { est la porosité }
\end{gathered}
$$

\section{IV.6 BIOTURBATION}

La bioturbation recouvre l'ensemble des processus de transformation du sédiment et de ses composés engendrés par la faune benthique. Selon leur mode de vie, et en particulier leur mode de nutrition, les organismes sont susceptibles de remanier le sédiment et de transporter des particules en profondeur (jusqu'à $5-15 \mathrm{~cm}$ ) depuis la surface, ou le contraire. Mais classiquement, l'action de bioturbation est représentée sous forme de mélange particulaire (biodiffusion) appliqué sur une épaisseur déterminée (variable selon les espèces) du sédiment superficiel. D'autre part, certains organismes sont reconnus pour ramollir le sédiment, d'autres au contraire pour le rigidifier, par exemple en construisant des tubes rigides à partir de particules sédimentaires cimentées par du mucus. La résultante de ces mécanismes est une modification de la nature du sédiment et de son érodabilité. Cependant leur prise en compte reste difficile car les effets sont rarement quantifiés et surtout la faune benthique montre une richesse spécifique dont le signal moyen est difficile à percevoir (e.g. Le Hir et al., 2007, [6]).

\section{D SYNTHESE SUR LES MODÈLES DE SÉDIMENT, EXEMPLES}

\section{V.1 APPROCHE CLASSIQUE : SÉDIMENT MULTICOUCHES}

Dans une majorité de problèmes (à l'exception notable des glissements) les transferts horizontaux sont négligeables, de telle sorte qu'un modèle de sédiment est formulé en mode unidimensionnel vertical, même s'il est discrétisé en 3D pour rendre compte des disparités horizontales qui découlent des conditions initiales ou des successions d'érosion/dépôt. Le sédiment est en général discrétisé en couches au sein desquelles des réactions physico-chimiques sont résolues. Les variables d'état sédimentaires sont la densité, la concentration totale ou un équivalent (indice des vides), ou depuis peu la concentration des différentes classes de particules considérées. Différents systèmes de coordonnées sont utilisés: cordonnées réelles ou « naturelles », coordonnées matérielles (en « $\mathrm{m}$ de sédiment $\mathrm{sec} »)$ ou encore coordonnées iso-densité (e.g. Sanchez, 1992). Par continuité, les teneurs en eau sont déduites ainsi que les transferts d'eau interstitielle. Parfois, la consolidation est traitée de façon simplifiée en affectant un taux de passage dans la couche inférieure en fonction de la concentration locale. Le choix du pas d'espace vertical est délicat, car les épisodes de dépôt sont en général répartis sur une durée assez longue, avec des quantités déposées très faibles à chaque pas de temps. La difficulté de la gestion des dépôts provient du risque de fréquemment mélanger des dépôts frais avec du sédiment consolidé, et ainsi d'accentuer numériquement l'effet de consolidation, ou alors de devoir multiplier le nombre de couches très fines, ce qui conduit à des contraintes numériques et des temps de calcul rédhibitoires. Enfin, à notre connaissance, aucun modèle ne considère les variations de porosité des sables sous l'action des vagues, malgré la forte dépendance des conditions de remaniement à la porosité. S'agissant des modèles diagénétiques, le nombre de couches considéré est généralement faible, et la discrétisation s'appuie sur des contrastes d'environnement: par exemple couche oxyque (typiquement 1 à $2 \mathrm{~cm}$ ) en surface, puis couche bioturbée, puis couche profonde (Fig. 5). Il faut relever que souvent les modèles de diagénèse utilisés supposent des flux de dépôt réguliers et donc des vitesses d'enfouissement prédéterminées, ce qui est incompatible avec un modèle sédimentaire de type processus.

Parmi les applications opérationnelles récentes, on peut mentionner la prise en compte de l'état consolidé du sédiment en morte eau dans un estuaire macrotidal, grâce à des couches de sédiment millimétriques, la simulation du changement saisonnier ou événementiel de la nature du sédiment superficiel (sable ou vase) à l'embouchure de la Seine (Waeles et al., 2006)[15], la prise en compte dans la modélisation écologique du dépôt et de la minéralisation de la matière détritique dans le sédiment, augmentant ainsi le temps de séjour des nutriments dans le système côtier (e.g. Cugier et al., 2005) [2].

\section{V.2 MODÉLISATION CONTINUE EAU/SÉDIMENT}

Dans certains cas (par exemple lorsque les concentrations en suspension et les dépôts sont très abondants) les gradients de concentration sont peu marqués et l'interface entre l'eau et le sédiment difficile à définir. Il est alors tentant d'inclure dans la colonne d'eau (plus ou moins chargée en particules) le sédiment de surface. C'est le concept de modélisation continue (e.g. Le Hir et Cayocca, 2002 [5]) : l'équation de quantité de mouvement est résolue dans le 
sédiment, à condition de prendre en compte la viscosité accrue voire un comportement laminaire non newtonien du sédiment, l'entravement de la vitesse de chute et l'amortissement des turbulences par les stratifications. Ce concept a été appliqué avec succès pour simuler la formation de la crème de vase dans l'estuaire de la Gironde, ou le glissement sous-marin de Nice en 1979 (Le Hir et Cayocca, $2002[5]$

\section{VI — DISCUSSION ET CONCLUSION}

La prise en compte du compartiment « sédiment» et sa modélisation sont d'abord motivées par le suivi au cours du temps de la disponibilité du sédiment et la caractérisation du sédiment superficiel : distribution granulométrique, rugosité de surface, présence de végétaux ou de zoobenthos. Lorsque le sédiment est non cohésif, l'accent doit être mis sur la porosité et la granulométrie, et si cette dernière est étendue, le pavage consécutif à un tri granulométrique dans une couche active doit être pris en compte. Lorsque le sédiment est cohésif, la concentration en vase du sédiment et particulièrement sa fraction argileuse sont déterminantes pour quantifier expérimentalement l'érodabilité. Si l'on dispose d'informations montrant une bonne stabilité temporelle de ces caractéristiques, il est possible de se passer de modèle sédiment. Dans le cas contraire, un modèle de sédiment multicouche doit être mis en place, et des publications récentes en ont montré la faisabilité, même dans le cas des mélanges sable/vase. Pour les sédiments vaseux, un module de consolidation est en principe obligatoire, sauf si les échanges instantanés par érosion/dépôt sont remplacés par une érosion ou un dépôt net sur un laps de temps assez long : cette pratique reste encore courante par souci d'économie de calcul, mais rarement justifiée.

Pour l'étude du devenir des composés biochimiques, de nombreux modèles de diagénèse précoce ont été développés, mais leur intégration dans des modèles sédimentaires finement discrétisés est rare : cela ne soulève pas de difficulté particulière, si ce n'est l'allongement des temps de calcul. Enfin la prise en compte du rôle des organismes, tant sur les échanges sédimentaires que sur les transformations chimiques dans le sédiment superficiel se heurte à l'extrême diversité des organismes et de leur comportement.

Il manque aujourd'hui un document-guide pour définir la modélisation minimaliste requise en fonction des questions d'environnement côtier à traiter : ce devrait être l'objet de travaux collaboratifs et pluridisciplinaires futurs.

\section{VII — REMERCIEMENTS}

Ce travail de synthèse a été réalisé dans le cadre de l'Action Transversale et Innovante du Programme National d'Environnement Côtier (PNEC) sur l'érodabilité des sédiments naturels. L'auteur remercie Françoise Andrieux et Annie Chapelle (IFREMER/ DYNECO/Pelagos) pour l'apport d'informations concernant les modèles biochimiques de sédiment.

\section{VIII — REFERENCES}

[1] Boudreau B.P. (1997). - Diagenetic models and their implementation. Modelling transport and reactions in aquatic sediments. Springer-Verlag Ed., 414 p.

[2] Cugier P., Ménesguen A., Guillaud J.F. (2005). - Threedimensional (3D) ecological modeling of the Bay of Seine (English Channel, France). Journal of Sea Research, 54 : 104 124.

[3] Le Hir P., Bassoullet P. \& L'Yavanc J. (1989). — New developments about mud transport models. Application to a macrotidal estuary. In (Ed. S.S.Y. Wang) « Sediment Transport Modeling », Proc. of the Int. Symp. New Orleans, 14-18 Aug., ASCE pub., 94-99.

[4] Le Hir P., Cann P., Jestin H. \& Bassoullet P. (2006). Instrumentation légère pour la mesure de l'érodabilité des sédiments vaseux ou sablo-vaseux. Compte-rendu des IXè journées nationales Génie Côtier - Génie Civil, L'Aber Wrac'h, 5 : 9 16.

[5] Le HiR P. \& CAYOCCA F. (2002). - Application of the continuous modelling concept to mud slides in open seas. In J.C. Winterwerp and mC. Kranenburg (eds) Fine Sediment Dynamics in the Marine Environment, Elsevier, proceedings in Marine Science, 5 : 545-562.

[6] Le Hir P., Monbet Y. \& Orvain F. (2007). - Sediment erodability in sediment transport modelling: can we account for biota effects ?Continental Shelf Research, 27 : 1116-1142.

[7] Merckelbach L. (2000). - Consolidation and strength evolution of soft mud layers. PhD thesis, Delft University of Technology, $151 \mathrm{p}$.

[8] Panagiotopoulos I., Voulgaris G. \& Collins M.B. (1997). - The influence of clay on the threshold of movement of fine sandy beds. Coastal Engineering, 32 : 19-43.

[9] Pham Van Bang D., Lefrançois E., Sergent P. \& Bertrand F. (2006). - Approche expérimentale par IRM et modélisation de la sedimentation et de la consolidation de la vase. Compterendu des IXè journées nationales Génie Côtier - Génie Civil, L'Aber Wrac'h, 4 : 9-17.

[10] Silva Jacinto R. \& Le Hir P. (2001). — Response of stratified muddy beds to water waves. In W.H. McAnally and A.J. Mehta (eds) Coastal and Estuarine Fine Sediments Processes, Elsevier, proceedings in Marine Science, 3 : 95-108.

[11] Slomp C.P., Epping E.H.G., Helder W. \& Van Rassphorst W. (1996). - A key role for iron-bound phosphorus in authigenic apatite formation in North Atlantic continental platform sediments. Journal of marine Research, 54 : 1179-1205.

[12] Toorman E. (1996). - Sedimentation and self-weight consolidation: general unifying theory. Geotechnique, 46(1) : 103-113.

[13] VAN Ledden M., VAN Kesteren W.G.M. \& WinterWerp J.C. (2004). - A conceptual framework for the erosion behaviour of san-mud mixtures. Continental Shelf Research, 24-1 : 1-11.

[14] WinterwerP J. \& VAN Kesteren W. (2004). — Introduction to the physics of cohesive sediment in the marine environment. Elsevier, 466 p. + annexes.

[15] Waeles B., Le Hir P., Lesueur P. \& Delsinne N. (2007). Modelling sand/mud transport and morphodynamics in the Seine river mouth (France): an attempt by using a processbased approach. Hydrobiologia (in press). 